Recepción: 01 / 03/ 2018

Aceptación: 15 / 05 / 2018

Publicación: 07 / 08 / 2018

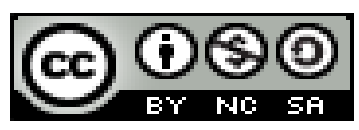

Ciencias de la Educación

Artículo Científico

\title{
Instrumentos de evaluación formativa para el perfeccionamiento de la producción escrita del idioma inglés
}

Instruments of formative evaluation for the improvement of the written production of the english language

Instrumentos de avaliação formativa para a melhoria da produção escrita da língua inglesa

Dorys Maribel Cumbe Coraizaca ${ }^{\mathrm{I}}$

dm.cumbe@uta.edu.ec

Sandra Elizabeth Hidalgo Agualongo II

sehidalgo4@espe.edu.ec

Ana Jacqueline Guamaní Aymacaña III

ana.guamani9183@utc.edu.ec

Correspondencia: $\underline{\text { dm.cumbe@ uta.edu.ec }}$

I. Docente de la Universidad Técnica de Ambato, Facultad de Ciencias Humanas y de la Educación, Ambato, Ecuador.

II. Docente de la Universidad de las Fuerzas Armadas ESPE, Centro de Idiomas, La Tacunga, Ecuador.

III. Docente de la Universidad Técnica de Cotopaxi, Ambato, Ecuador. 


\section{Resumen}

Con el paso del tiempo la metodología del idioma extranjero ha venido experimentando cambios, que no solo tienen que ver con la utilización de medios digitales, sino métodos fáciles de aplicar con herramientas accesibles por parte de los estudiantes y los docentes. En el presente artículo se desea determinar si los instrumentos de evaluación formativa que son herramientas que ayudan a documentar el desempeño del estudiante, tales como portafolios, ensayos, cuentos tienen relación con la producción escrita, que es la habilidad para desarrollar las capacidades de organización, estructuración y distribución de ideas del idioma inglés. La metodología que se utilizó fue cualicuantitativa debido a que se utilizó información numérica y características empíricas de las variables en estudio. Mediante una encuesta a los docentes y estudiantes de la Unidad Educativa Luis A. Martínez de tercer año de Bachillerato General Unificado (BGU), se determino que no utilizan los instrumentos de evaluación formativa y que la producción escrita no es la adecuada para el nivel de inglés en el que se encuentran. Se contrasto la hipótesis atraves del chi cuadrado, donde se pudo conocer que si existe relación entre las dos variables sin embargo no existe su aplicación en la unidad educativa, por lo que se concluye que es necesario socializar de una manera mas eficiente estos instrumentos y se abarque todo el funcionamiento de una manera muy detallada a través de capacitaciones y jornadas que ayude al docente a entender y manejar claramente estos instrumentos que generarían mayor eficiencia en la producción escrita de los estudiantes.

Palabras clave: Evaluación formativa, desempeño academico, instrumentación, perfeccionamiento de la escritura, idioma inglés. 


\begin{abstract}
Over time, the methodology of the foreign language has been undergoing changes, which not only have to do with the use of digital media, but easy methods to apply with accessible tools by students and teachers. In this article we want to determine if formative assessment tools are tools that help document student performance, such as portfolios, essays, stories are related to written production that is the ability to develop organizational skills, structuring and distribution of English language ideas. The methodology used was qualitative-quantitative because numerical information and characteristics of the variables under study were used. Through a survey for teachers and students of the Luis A. Martínez School of the third year of Unified General Baccalaureate, it was determined that formative evaluation instruments are not used and that written production is not adequate for the level of English in which they are. It is contrasted the hypothesis through the application of the chisquare it was possible to know that there is a relationship between the two variables, however, its application does not exist at Luis A. Martínez School, so it is necessary to socialize these instruments and explain the contribution they would generate and include the detailed operation through trainings which would help teachers to understand and manage the instruments clearly and would generate greatest efficiency in the written production of the students.
\end{abstract}

Keywords: formative evaluation, academic performance, improvement of the writing, english language 


\section{Introducción}

Los instrumentos de evaluación formativa son herramientas importantes para medir el desempeño del estudiante en clase en la habilidad escrita. Hay veces en que el docente solo se proyecta en desarrollar las habilidades del habla, lectura y de escucha, dejando de lado la escritura que es un complemento para las demás. Por esta razón, esta investigación se basa en determinar la relación de las dos variables y si se ha aplicado los instrumentos de evaluación formativa.

Como primer término es necesario conocer los principales conceptos que envuelven las dos variables. Por un lado, los instrumentos de evaluación formativa es el medio a través del cual se recaba y registra información que permita emitir una valoración, donde se hallan los posters, diarios, informes, entre otros. Y por otro, la producción escrita donde cada uno aprende sobre sí mismos y comunican sus percepciones del mundo que los rodea a otros.

Además, se define la metodología del artículo, describiendo la población y la muestra con que se va a trabajar, y el instrumento que se va a aplicar. Los Resultados por otro lado son necesarios para comprobar la hipótesis del documento y la discusión donde se realiza un análisis de los principales hallazgos en la investigación y su relación con otros estudios.

Y por último se concluye resaltando los principales aspectos que se describieron en el artículo.

\section{Desarrollo.}

Se define a los instrumentos de evaluación formativa como las herramientas que ayudan a documentar el desempeño del estudiante, para luego verificar los logros obtenidos y evaluar los 
productos elaborados. Además, permiten determinar si una persona es competente o no considerando las destrezas, actitudes, conocimientos, habilidades y valores puestas en juego en el ejercicio de una acción en un contexto determinado. De manera más clara “un instrumento de evaluación es el medio a través del cual recabamos información y registramos los datos que nos van a permitir emitir una valoración” (López, 2009, p. 66).

Es importante recalcar que un instrumento de evaluación no debe estar enfocado al aprendizaje memorístico, como por ejemplo los exámenes, por lo que se caería en el tradicionalismo y a su vez se provocaría un proceso adaptativo de los estudiantes para el tipo de evaluación que se va a utilizar, en vez de conseguir el mejor aprendizaje utilizando varias técnicas e instrumentos de evaluación formativa. Sanmartí, (2007) afirma que "los instrumentos de evaluación orientan el proceso de toma de decisiones que permite mejorar y desarrollar con éxito los procesos de enseñanza y aprendizaje". En otras palabras, la búsqueda y la obtención del aprendizaje surgen de procesos de evaluación y a través de ellos podemos modificar y tomar decisiones que nos conduzcan a docentes y alumnos, a nuevas situaciones de evaluación y aprendizaje.

Instrumentos de evaluación formativa con predominio en la expresión escrita

La producción escrita ha terminado siendo la forma más usual de evidenciar los conocimientos, esto se debe al elevado número de estudiantes, a la posibilidad de corregir los trabajos una vez se han realizado y a la evidencia física que queda para posibles reclamaciones. (Ferreras \& González, 2006)

Las posibilidades son muy extensas, y las opciones a los formatos habituales también. Por ejemplo, lo usual es que el examen sea una prueba individual, pero también puede haber exámenes 
conjuntos, al igual que los trabajos y otros modelos de expresión escrita. López V. (2009) menciona los instrumentos más habituales en la escritura y sus características en una evaluación formativa:

\section{Gráfico $\mathrm{N}^{\circ}$ 1: Instrumentos de evaluación formativa}

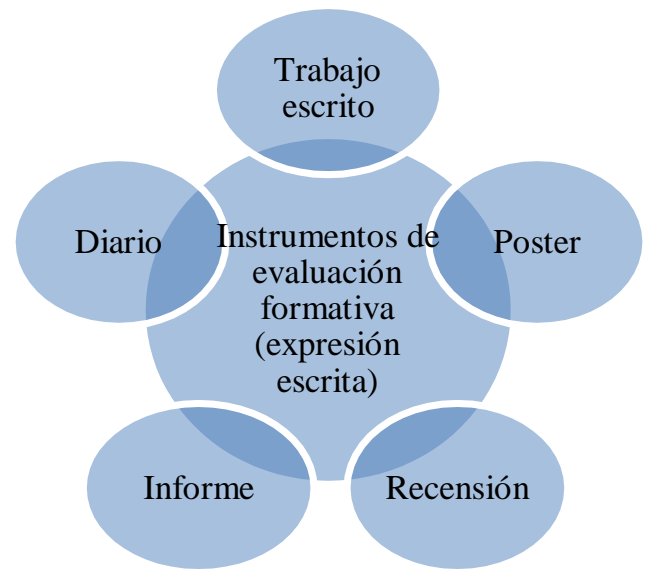

Fuente: Cabrera, F (2000) “Evaluación formativa” Madrid, España. Síntesis Educación.

\section{Instrumentos recopiladores en la escritura}

López, V. (2009), menciona tres importantes recopiladores en la expresión escrita en el proceso de enseñanza-aprendizaje.

- Portafolio: es un instrumento en el cual se realiza una selección de evidencias sobre un proceso de aprendizaje que contiene sólo aquello que es considerado importante.

- Carpeta: es un conjunto de documentos que suelen ser todos los que se tienen de una asignatura y que se suponen la totalidad de la documentación utilizada en un curso.

- Memoria: Es la disertación sobre alguna materia, que se realiza al final del acontecimiento y que sirve de recapitulación, contiene hechos y contribuciones de información que está relacionada y que ilustra lo acontecido. 
Producción escrita

Hay autores que definen a producción escrita desde diferentes puntos de vista. Por ejemplo, Ong (1990) menciona lo siguiente "la escritura, la más trascendental de todas las invenciones tecnológicas humanas” (p.87), nació hace relativamente poco, hacia el 3500 a.C. Pero pronto se convirtió en una potente "tecnología de intelecto" Goody (1968, p.11) que facilito "la domesticación de la mente salvaje" Goody (1977). En otras palabras, la escritura fue una creación humana que terminó moldeando muchas comunidades y también la mismísima mente de su creador.

En conclusión, la acción de escribir está considerada como un poderoso instrumento de reflexión, en el cual, los redactores aprenden sobre sí mismos y comunican sus percepciones del mundo que los rodea a otros. Además, el acto de escribir se materializa en un proceso complejo en el cual el redactor imagina a la audiencia, desarrolla ideas, produce anotaciones, borradores y un texto elaborado con el fin de satisfacer a la audiencia.

\section{Metodología.}

El enfoque de la presente investigación es de carácter cuali-cuantitativo. Porque se analizó e interpretó el problema a investigarse, contextualizando y asumiendo una realidad dinámica en el campo educativo mediante una medición controlada. Se trabajó con la investigación de campo al desarrollarse la investigación en el lugar donde se suscitan los hechos, es decir en la Unidad Educativa Luis A. Martínez. Y de tipo bibliográfico porque se recogió información teórica que ayudó a profundizar la comprensión de las dos variables mediante criterios de varios autores. 
Tabla $N^{\circ}$ 1: Población

\begin{tabular}{|c|c|c|}
\hline POBLACIÓN & CANTIDAD & $\%$ \\
\hline ESTUDIANTES & 141 & 98 \\
\hline DOCENTES & 3 & 2 \\
\hline TOTAL & 144 & 100 \\
\hline
\end{tabular}

Fuente: Unidad Educativa Luis A. Martínez

Elaborado por: Tibán, F. (2015)

La investigación se realizó con los estudiantes del Tercer Año de Bachillerato General Unificado, de la Unidad Educativa Luis A. Martínez de la ciudad de Ambato provincia de Tungurahua. La población involucrada en el problema objeto de estudio fueron: 141 estudiantes y 3 docentes del área de inglés.

\section{Resultados.}

Realizando un análisis minucioso de la información obtenida a través de las preguntas más importantes de la encuesta donde se solicitó datos acerca de la frecuencia de actividades escritas; aplicación de portafolios, ensayos cortos, diarios; técnicas de producción escrita; y actividades de composición libre, se pudo realizar la correlación de las dos variables objeto de estudio donde están especificadas las frecuencias observadas y frecuencias esperadas.

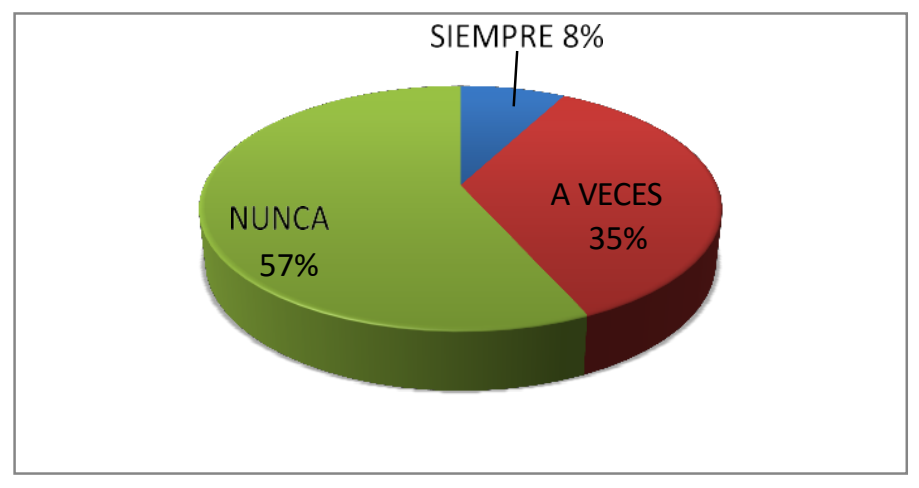




\section{¿En las clases de inglés, se aplica la elaboración de portafolios, ensayos}

\section{cortos, diarios, posters, como instrumentos de evaluación escrita?}

De acuerdo a los datos obtenidos, el $57 \%$ que corresponde a 80 estudiantes manifestaron que el docente NUNCA aplica la elaboración de portafolios, ensayos cortos, diarios, posters, como instrumentos de evaluación escrita, el 35\% que pertenece a 50 estudiantes expresaron que NUNCA, y el 8\% que comprende a 11 estudiantes dijeron que SIEMPRE. Evidentemente, en las clases de inglés, el docente no utiliza instrumentos de evaluación formativa que ayuden al desarrollo de la producción escrita del idioma. Es necesario utilizar varios instrumentos de evaluación para evitar el tradicionalismo con unas clases monótonas.

\section{¿En las clases de inglés, se realiza actividades escritas de composición libre}

como cuentos, historias, diarios personales, e-mails, etc.?

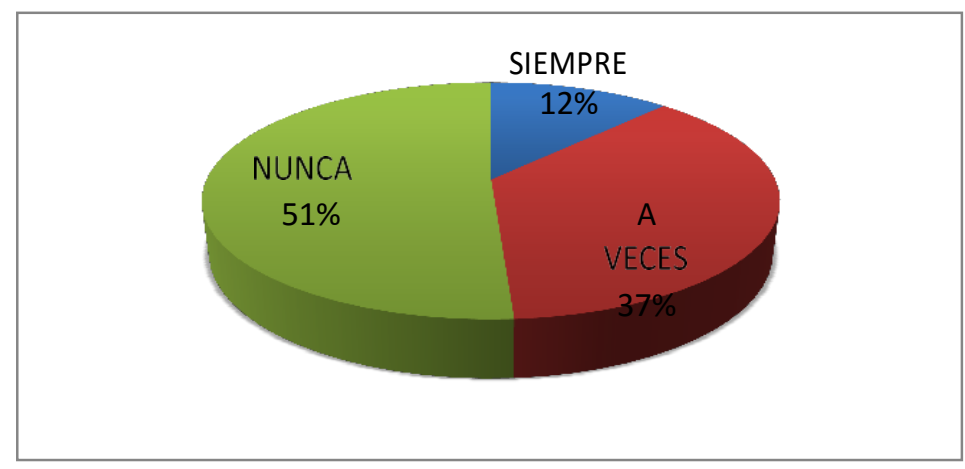

De acuerdo a los datos obtenidos, el $51 \%$ que comprende a 72 estudiantes manifestaron que NUNCA se realiza actividades escritas de composición libre, mientras que el 37\% que pertenece a 52 estudiantes dijeron que A VECES, y el 12\% que corresponde a 14 estudiantes mencionaron SIEMPRE. Evidentemente, en las clases de inglés, el docente no realiza actividades de composición libre como cuentos, historias, diarios personales, e-mails, etc., esto provoca que el estudiante no elabore escritos de su tema de interés, ocasionando un poco de frustración. El equilibrio de diferentes 
actividades escritas, tanto controladas como libres, ayuda al desarrollo de la destreza productiva de la escritura del idioma inglés.

Para comprobar la hipótesis de la influencia de los instrumentos de evaluación formativa en la producción escrita del idioma inglés se debe demostrar que el valor calculado $\left(\mathbf{X}^{2} \mathbf{C}\right)$ sea mayor al valor tabulado $\left(\mathbf{X}^{2} \mathrm{t}\right)$ donde:

$\mathbf{X}^{2} \mathbf{C}=33,8205$

$\mathbf{X}^{2} \mathrm{t}=12.5916$

“Los Instrumentos de evaluación formativa SI influyen en la producción escrita del idioma inglés en los estudiantes del Tercer Año de Bachillerato General Unificado, de la Unidad Educativa Luis A. Martínez de la ciudad de Ambato provincia de Tungurahua”

\section{Gráfico $\mathbf{N}^{\circ}$ 2: Aceptación de la hipótesis}

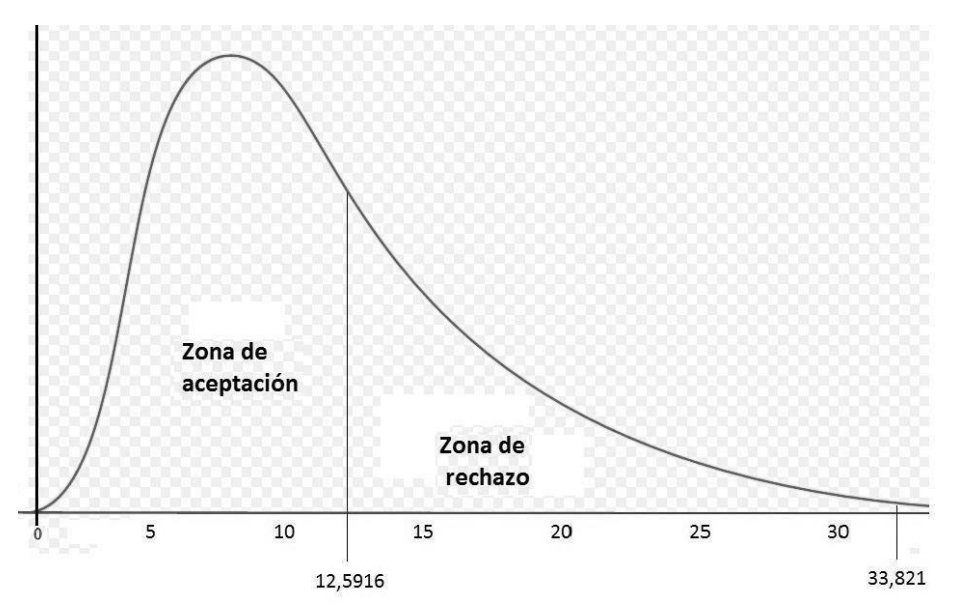




\section{Discusión.}

Una manera muy acertada de medir el desempeño escolar es a través de la presentación del portafolio de trabajos escritos desarrollados en clase o en casa. Mucho se ha hablado de que la única forma de medir ese esfuerzo era con las evaluaciones escritas, sin embargo, con la aparición de instrumentos de evaluación formativa se ha mejorado la metodología de enseñanza docenteestudiante. Si bien es cierto, el docente presenta más trabajo, el alumno puede practicar más su escritura y convertirla en un conocimiento a largo plazo. Tanto los instrumentos de evaluación formativa junto con la producción escrita están relacionados de manera muy importante, de acuerdo a la prueba del chi cuadrado. Sin embargo, el examen escrito continúa siendo el único instrumento para evaluar el conocimiento del idioma extranjero, descartando instrumentos valiosísimos como los portafolios, ensayos, posters como método de evaluación escrita, así como actividades escritas de composición libre (historias, cuentos, diarios).

En el trabajo investigativo de Vázquez, (2012), constató que existe una diversidad amplia de instrumentos para utilizar en el proceso de evaluación formativa, de manera que, además, de servir como diagnóstico de lo aprendido, se ha manejado como una retroalimentación para el proceso de aprendizaje. El investigador destaca el portafolio de evidencias como un gran instrumento y manifiesta que es un gran recurso de evaluación para realizar comparaciones de los progresos con los niños y de forma grupal, al mismo tiempo ayuda a dar una escala de calificación con los reportes sistemáticos en la evaluación continua. 


\section{Conclusiones.}

- Herramientas como: ensayos cortos, diarios, posters, portafolios, historias, permiten involucrar al estudiante en el proceso de aprendizaje de una manera más activa y comprometida.

- Los instrumentos de evaluación formativa no son aplicados en la clase, generando un desbalance en el aprendizaje del estudiante y el nivel de estudio en el que están. El examen escrito continúa siendo el instrumento más utilizado, impidiendo desarrollar la destreza escrita del estudiante que evita alcanzar un aprendizaje significativo.

- A través de la aplicación de la prueba del chi cuadrado se pudo determinar la relación directa entre los instrumentos de evaluación formativa y la producción escrita del idioma inglés y se llegó a la conclusión de que los estudiantes no desarrollan la destreza escrita acorde los estándares internacionales.

\section{Bibliografía.}

Cabrera, F. (2000). Evaluación de la formación. Madrid: Síntesis Educación.

Ferreras, V., \& González, A. (2006). Evaluación para la mejora de los centros docentes. Madrid; España: Wolters Kluwer.

López, V. (2009). Evaluación Formativa y Compartida en Educación Superior. Madrid, España: Narcea S.A.

Sanmartí, N. (2007). 10 ideas claves: evaluar para aprender. Madrid, España: Graó.

Troya, S. (2007). Módulo de Diseño Curricular . Madrid, España.

Valero, J. (2000). Educación persnalizada. México D.F, México: Progreso S.A. 